\title{
The Salt Swap intervention to reduce salt intake in people with high blood pressure: protocol for a feasibility randomised controlled trial
}

Sarah Payne Riches ${ }^{1 *}$ (D), Carmen Piernas ${ }^{1}$, Paul Aveyard ${ }^{1}$, James P. Sheppard ${ }^{1}$, Mike Rayner ${ }^{2}$ and Susan A. Jebb ${ }^{1}$

\begin{abstract}
Background: High salt intake is a risk factor for hypertension and cardiovascular disease. Reducing salt intake has been shown to reduce blood pressure. Despite population-level interventions, including product reformulation and public awareness campaigns, adult salt consumption in the UK still exceeds recommendations; this is primarily due to salt consumed in processed and pre-packaged foods. Moderate or high-intensity dietary advice to encourage individuals to reduce their salt intake has been shown to be effective at reducing blood pressure, but evidence of the effectiveness of interventions that are suitable for delivery at scale in routine primary care is scarce. This feasibility trial investigates a complex behavioural change intervention to reduce dietary salt intake and blood pressure by encouraging individuals to purchase lower-salt foods when grocery shopping.
\end{abstract}

Methods: This randomised controlled trial will test the feasibility of a novel intervention to reduce salt intake, and the trial procedures to assess its effectiveness. We will recruit participants through UK general practices and randomise 40 participants with high blood pressure, in a 2:1 allocation to receive either the Salt Swap intervention or a control information leaflet. The primary outcomes relate to the criteria for progression to a large-scale trial. These include follow-up rates at 6 weeks, fidelity of intervention delivery and use of the intervention mobile app. Secondary outcomes include the effect of the intervention on the salt content of purchased foods (grams per $100 \mathrm{~g}$ ), urinary sodium excretion assessed through 24-hour urine samples and blood pressure. Trial process measures will be collected and qualitative assessment will provide insights into participant engagement with the intervention content and perceived barriers to and facilitators of salt reduction dietary behavioural change.

Discussion: If the outcomes indicate the trial is feasible and there is evidence that behavioural change may result in salt reduction, we will proceed to a definitive trial to test the effectiveness of the intervention to lower blood pressure. If successful, this intervention approach could be applied not only to people with high blood pressure, but also to the wider population with normal blood pressure in whom dietary salt intake exceeds recommendations.

Trial registration: ISRCTN, 20910962. Registered on 5 April 2017.

Keywords: Salt intake, Urinary sodium, Blood pressure, Food purchasing, Behaviour change

\footnotetext{
* Correspondence: sarah.payneriches@phc.ox.ac.uk

${ }^{1}$ Nuffield Department of Primary Care Health Sciences, University of Oxford

Radcliffe Observatory Quarter, Woodstock Road, Oxford OX2 6GG, UK

Full list of author information is available at the end of the article
}

(c) The Author(s). 2019 Open Access This article is distributed under the terms of the Creative Commons Attribution 4.0 International License (http://creativecommons.org/licenses/by/4.0/), which permits unrestricted use, distribution, and reproduction in any medium, provided you give appropriate credit to the original author(s) and the source, provide a link to the Creative Commons license, and indicate if changes were made. The Creative Commons Public Domain Dedication waiver (http://creativecommons.org/publicdomain/zero/1.0/) applies to the data made available in this article, unless otherwise stated. 


\section{Background}

Cardiovascular disease (CVD) is the leading cause of premature mortality globally and a significant contributor to preventable morbidity [1-4]. Hypertension is one of the most significant risk factors for CVD, and a key contributor to the global burden of disease [5, 6]. At least one quarter of adults in the UK (and more than half of those older than 60 years) have hypertension [7]. There is a positive association between blood pressure and dietary salt intake [8], both in individuals with hypertension and also in those with normal blood pressure [9]. Despite UK government recommendations for adults to reduce dietary salt intake to $<6 \mathrm{~g} /$ day, intake remains high at an average of $8.1 \mathrm{~g} /$ day [10], with around three quarters of this derived from packaged or processed foods [11]. Overall, 70\% of the adult population are estimated to have salt intake $>6 \mathrm{~g} /$ day [10].

Clinical trials have proven that dietary interventions with intensive support to reduce salt intake, sometimes involving the provision of low-salt foods, can significantly reduce systolic blood pressure, by up to $7 \mathrm{mmHg}$ [12-14]. In addition, systematic reviews have demonstrated that advice-based interventions such as nutritional counselling or tailored education can be effective $[15,16]$, but the effectiveness of these interventions depends largely on intensity. Interventions most likely to be effective are those including moderate-to-high-intensity advice, involving multiple one-to-one or group counselling sessions [17]. The challenge is that such interventions are not feasible to deliver at scale by non-specialist staff without a significant expansion in the workforce and training. At present any dietary advice in routine primary care usually consists of non-tailored information to help patients maintain a lowsalt diet [7]. There is a wide range of salt content in foods within and between product categories, and it is difficult for non-specialists to provide accurate and personalised advice to help individuals achieve a low-salt diet.

Brief behavioural interventions have been used successfully in primary care to encourage smoking cessation [18] and reductions in alcohol intake [19] but evidence on the effectiveness of brief interventions for dietary change is limited. A recent pilot study of a low-intensity dietary counselling intervention to reduce blood cholesterol demonstrated a significant beneficial short-term effect [20], indicating potential for a brief-intervention approach. However, a study of a brief intervention to reduce blood pressure, covering a range of strategies including diet, showed no effect [21], and there is currently no established brief intervention known to be effective to reduce salt intake.

One approach to magnify the effectiveness of advicebased interventions for dietary change is to use technology to deliver personalised advice at the point at which individuals make a choice about the food and drink they buy or consume. Studies using digital tools such as smartphone applications (apps) as a standalone intervention to reduce salt intake indicate their potential effectiveness $[22,23]$.

This study investigates the feasibility of a trial examining a complex, behaviour change intervention to reduce salt intake, combining brief advice delivered by non-specialist health practitioners in primary care with use of an app to guide food-purchasing decisions. It aims to motivate and support individuals with high blood pressure to reduce their salt intake by swapping to lower-salt products when grocery shopping. A definitive trial to show whether this intervention is effective at lowering salt intake and blood pressure would need to be large and would be expensive. Before this is justified, we need to ensure that people engage with the intervention, that it can reduce salt intake in the short term and that the research methods run as expected.

\section{Objectives}

The primary aim of this study is to test the feasibility of a randomised controlled trial (RCT) to study the effectiveness of this complex behavioural intervention to lower salt intake in people with raised blood pressure. The primary objectives are therefore to assess the criteria for progression to a full trial, which would examine the effect on blood pressure. The primary objectives will assess the:

- Rate of attendance at the follow-up session

- Proportion of the essential intervention elements that are delivered in the intervention session

- Proportion of participants using the Salt Swap app

Secondary objectives are to:

- Gain an indication of effectiveness of the intervention in terms of salt intake, salt content of purchased foods and blood pressure at follow-up

- Understand the barriers and facilitators of successful engagement with the intervention

We will also test the trial procedures through a range of process measures.

\section{Methods}

\section{Trial design and setting}

This trial will be an individually randomised, parallel, two-arm, controlled feasibility trial with a 2:1 (intervention to control) allocation ratio. The primary outcome will be the criteria to progress to a full trial. Process evaluation and analysis of effectiveness outcomes may inform a future trial. Participants will be recruited through five primary care practices in Oxfordshire, UK. 


\section{Recruitment}

Practices will search the electronic health records of registered patients to find people who meet the age and blood pressure inclusion criteria. Prior to sending out the invitation letters, the general practitioner (GP) will screen the search results to ensure that all those identified are medically appropriate to participate in the trial and meet the clinical inclusion criteria. The practice will send potential participants an invitation letter asking them to contact the research team if they are interested in participating; the letter will also include a link to an electronic version of the participant information sheet. GPs may also identify eligible patients opportunistically during routine consultations or via the National Health Service (NHS) Health Check programme. We will aim to randomise 40 participants in the study.

\section{Eligibility criteria Inclusion criteria}

The inclusion criteria are:

- Most recent systolic blood pressure reading in the past 2 years $>130 \mathrm{mmHg}$ if they are currently taking anti-hypertensive medication or $>140 \mathrm{mmHg}$ if non-medicated

- If on pharmacological treatment for hypertension, the participant has been prescribed a stable dose for at least 6 weeks

- Willing and able to give informed consent for participation in the study

- Male or female, aged between 18 and 80 years

- English speaking

- Regularly shop in a supermarket (excluding online supermarkets), spending at least $£ 25$ at least once a fortnight

- Own a smartphone (android or iOS) and prepared to use an app for healthy eating

- Express a desire for support to improve the nutritional quality of their diet to reduce their CVD risk

\section{Exclusion criteria}

The exclusion criteria are:

- Currently on a clinician-supervised diet or a restricted diet

- Currently using or have experience of using the Foodswitch or Saltswitch apps

- Unable to read and understand the instructions provided in English

- Have secondary, previous accelerated or malignant hypertension documented in the electronic patient record

- Currently being assessed for diagnosis of hypertension
- Currently on any medication that may lead to hyponatraemia or fluid retention

- Existing or recent cardiovascular conditions: heart attack or stroke within the last 3 months, heart failure of New York Heart Association grade II and more severe or prolonged QT syndrome, angina, arrhythmia or atrial fibrillation

- Currently participating in another research study

- Considered by the GP as not suitable for or unable to meet the demands of the study or unlikely to comply with study procedures as stated in the protocol

- Planning to be away from home for more than 2 weeks consecutively during the 6-week intervention period

\section{Intervention and comparator Intervention}

This study investigates the Salt Swap intervention, which aims to help people swap to lower-salt alternatives for everyday foods when grocery shopping. The intervention development was informed by two established behaviour change frameworks, the Behaviour Change Wheel [24] and the Theoretical Domains Framework (TDF) [25]. These theoretical frameworks were used to identify the key determinants of behaviour, particularly the aspects of capability, opportunity and motivation, that need to be influenced in order to support people to reduce purchases of high-salt foods and drinks. The intervention development is described elsewhere.

The intervention incorporates motivation and education, problem solving, goal setting and action planning. It comprises two main components: brief advice provided by a healthcare professional (HCP) at the GP practice and instructions to use the Salt Swap app. Healthcare professionals, typically practice nurses or healthcare assistants, will be trained by one face-to-face training session with a researcher and by video-based training materials. The content of the 20-min one-toone advice session is based on best practice guidance and information by expert sources including NHS Choices and the British Heart Foundation (BHF) [26, 27]. It is supported by the Salt Swap booklet given to participants during the intervention visit.

This advice incorporates information on:

- The impact of salt on health and blood pressure in particular

- The health benefits of salt reduction

- Recommended salt intake

- Advice for cutting salt intake

- Examples of lower-salt alternatives

- Salt reduction goal setting

- Self-assessment

- Action planning to reduce salt intake 
In addition, during this session participants are encouraged to download a novel mobile phone app, Salt Swap, which delivers tailored, salient information on the salt content of products. Users scan a product barcode to receive nutritional information, presented using the traffic light labelling system recommend for use in the UK [28], and are shown suggested lowersalt alternatives. The app contains a database of food and drinks available in UK supermarkets and their nutritional composition. The app allows users to set goals for salt reduction and to receive feedback on the achievement of these goals and the salt reduction achieved through swapping to lower-salt products (see Fig. 1). All other clinical care will continue as usual.

\section{Control (usual care)}

Participants randomised to the control arm will receive a copy of the publicly available BHF Cut Down on Salt booklet [27] by post, or any revised version of this publication. On completion of their follow-up visit, i.e. when they have completed their participation in the study, they will be sent the same written advice that is given to intervention participants. All other clinical care will continue as usual.

\section{Participant flow}

Figure 2 illustrates the trial flowchart.

\section{Screening and informed consent}

Participants who are interested in taking part will contact the research team. A researcher will assess eligibility based on the full inclusion and exclusion criteria, verbally provide information as described in the participant information sheet and answer any questions about participation in the study. Those who are eligible will be booked into an initial face-to-face baseline study visit at their GP practice. When the participant attends the baseline visit, the researcher will provide verbal and written information about the trial and seek informed consent (see Table 1 for the schedule of procedures). Having had a chance to ask questions, those individuals willing to participate will give written informed consent by means of a participant-dated signature. The consent form will also be dated and signed by the researcher who presented and obtained the informed consent.

\section{Baseline}

The baseline assessment will be undertaken at the initial baseline visit following informed consent. The researcher will collect clinical measures of height (metres), weight

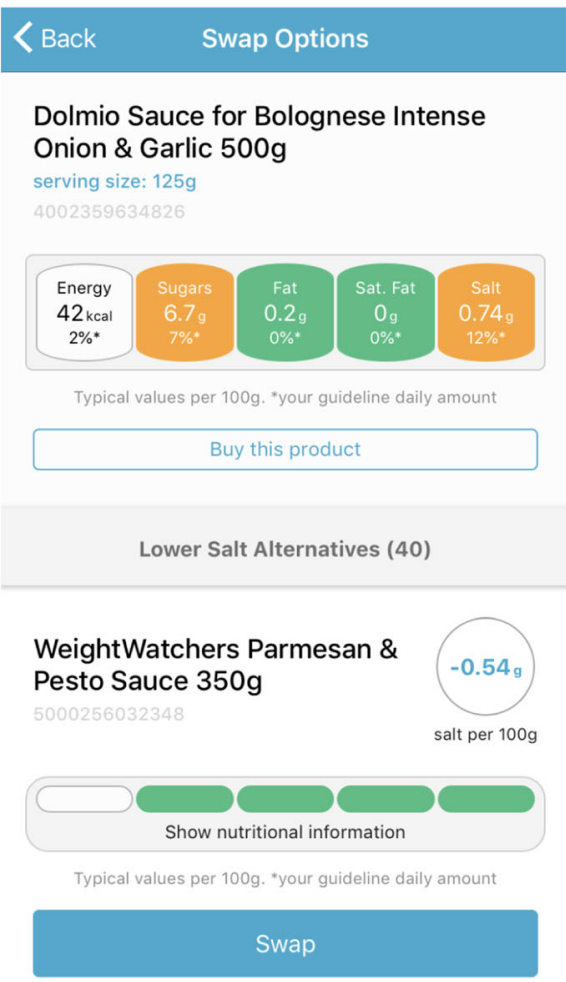

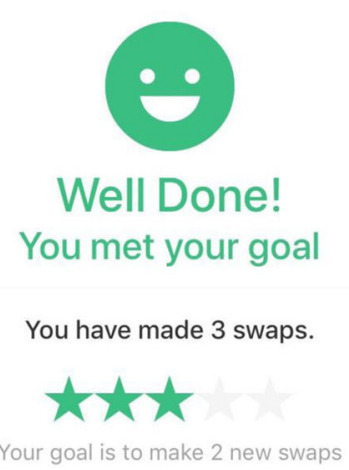

You have reduced your salt by $59.4 \%$

Fig. 1 Salt Swap app 
Table 1 Schedule of study visits, assessment and procedures

\begin{tabular}{|c|c|c|c|c|c|c|}
\hline & $\begin{array}{l}\text { Screening } \\
\text { phone call }\end{array}$ & $\begin{array}{l}\text { Enrolment and } \\
\text { baseline } \\
\text { (Visit 1) }\end{array}$ & Allocation & $\begin{array}{l}\text { Intervention } \\
\text { (Visit 2) }\end{array}$ & $\begin{array}{l}\text { Accompanied } \\
\text { shop } \\
\text { (Visit 3) }\end{array}$ & $\begin{array}{l}\text { Followup } \\
\text { (Visit 4) }\end{array}$ \\
\hline Time point (weeks from baseline) & -1 & 0 & 2 & $2-3$ & $4-9$ & $9-10$ \\
\hline Responsibility & $\begin{array}{l}\text { Central research } \\
\text { team }\end{array}$ & $\begin{array}{l}\text { Central research } \\
\text { team }\end{array}$ & $\begin{array}{l}\text { Independent } \\
\text { researcher }\end{array}$ & $\begin{array}{l}\text { Healthcare } \\
\text { professional }\end{array}$ & $\begin{array}{l}\text { Central research } \\
\text { team }\end{array}$ & $\begin{array}{l}\text { Central research } \\
\text { team }\end{array}$ \\
\hline \multicolumn{7}{|l|}{ STUDY PROCEDURES } \\
\hline Eligibility assessment & $\checkmark$ & & & & & \\
\hline Informed consent & & $\checkmark$ & & & & \\
\hline Baseline assessment including: & & $\checkmark$ & & & & \\
\hline Blood pressure & & $\checkmark$ & & & & \\
\hline Height and weight & & $\checkmark$ & & & & \\
\hline $\begin{array}{l}\text { Medical history and } \\
\text { medications }\end{array}$ & & $\checkmark$ & & & & \\
\hline $\begin{array}{l}\text { Shopping and diet } \\
\text { questionnaire }\end{array}$ & & $\checkmark$ & & & & \\
\hline $\begin{array}{l}\text { Instruction on baseline run-in } \\
\text { data collection }\end{array}$ & & $\checkmark$ & & & & \\
\hline Urine sample collection & & $\begin{array}{l}\checkmark(+ \text { up to } 2 \\
\text { weeks) }\end{array}$ & & & & \\
\hline Randomisation & & & $\checkmark$ & & & \\
\hline Intervention delivery & & & & $\checkmark$ & & \\
\hline Accompanied shop and interview & & & & & $\checkmark$ & \\
\hline $\begin{array}{l}\text { Follow-up outcome measures } \\
\text { including: }\end{array}$ & & & & & & $\checkmark$ \\
\hline Blood pressure & & & & & & $\checkmark$ \\
\hline $\begin{array}{l}\text { Medical history and medication } \\
\text { review }\end{array}$ & & & & & & $\checkmark$ \\
\hline $\begin{array}{l}\text { Intervention acceptability } \\
\text { questionnaire }\end{array}$ & & & & & & $\checkmark$ \\
\hline Urine sample collection & & & & & & $\begin{array}{l}\checkmark \\
\checkmark \\
\text { (days) }\end{array}$ \\
\hline
\end{tabular}

(kilograms), and blood pressure (seated systolic and diastolic blood pressure, measured after 5 min rest and using the mean of the second and third of three readings taken $1 \mathrm{~min}$ apart using a validated digital automatic blood pressure monitor, the OMRON M10-IT). The researcher will also record demographic information, relevant medication history and medications (verified through the patient's electronic clinical record) and details of the participant's shopping behaviour including the frequency of shopping, the number of people for whom they are shopping, the types of stores or settings most commonly visited for groceries and key influences on purchasing decisions (using an investigator-designed questionnaire).

At the end of the baseline visit participants will be instructed on how to use a mobile phone app to record their grocery shopping throughout the study. The app, called ShopScanner, is linked to a food and nutrient information database to enable recording of the nutrient content of purchased foods. They will also be asked to collect their grocery shopping receipts throughout the study.

\section{Baseline run-in period}

The baseline period will run for 2 weeks following the baseline assessment visit. Participants will be asked to collect a 24-hour urine sample within this baseline period, for the measurement of urinary sodium excretion (mmol/24-hour). They will also be asked to submit their grocery shopping till receipts for this period to the research team. Participants who return partial baseline 


\section{Participant identification}

Search conducted by GP practice and letters sent to patients

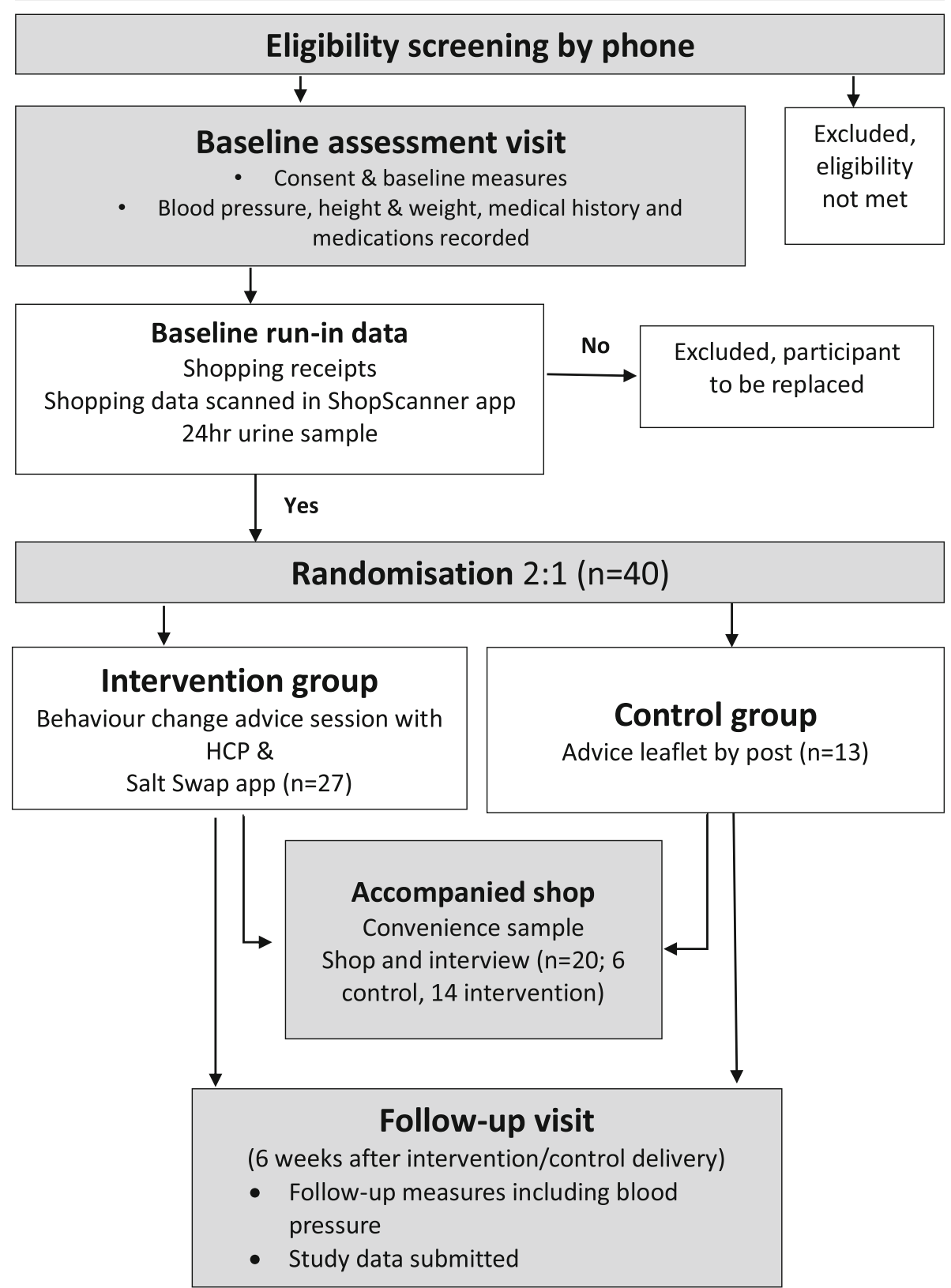

Fig. 2 Participant flow. GP, general practitioner; HCP, healthcare professional

data will be prompted, by phone or email, to return all requested data.

Participants will be randomised once the urine sample and baseline shopping data for the baseline period have been provided. Participants who do not complete these measures will not be randomised. Participants randomised to the intervention group will be booked to attend an intervention session with a HCP at their GP practice.
Control participants will receive the control intervention leaflet by post.

\section{Intervention visit}

The intervention will be delivered by a HCP at the GP surgery. Designated HCPs from the surgery will be specifically trained to deliver the intervention and 
to complete an intervention checklist. This visit will last $20-30 \mathrm{~min}$.

\section{Follow-up}

All participants will be invited to attend one follow-up assessment 6 weeks after they receive their allocated intervention (either the intervention visit or the control information sent by post). During this visit, a researcher will measure blood pressure and document any changes to relevant medical history or medications. Participants will be asked to complete an investigator-designed questionnaire to assess aspects of the intervention (or control leaflet) they received.

The follow-up questionnaire will collect data on changes in knowledge about dietary salt intake, awareness of salt intake and dietary-salt-related behaviours. Separate questions for each group will assess how helpful the Salt Swap intervention (advice session and Salt Swap app) or control leaflet were (1-5 scale from very unhelpful to very helpful) as well as the specific reasons why it was considered helpful or unhelpful (for the Salt Swap intervention only). The intervention group questionnaire will also collect data on app acceptability ("would you be interested in using a free app like the Salt Swap app if it was publically available in future?") and frequency of use (always, most of the time, occasionally, never). Both groups will be asked if any other forms of support (e.g. apps, tools, advice or contact with health professionals) were used throughout the study.

Participants will be asked to submit their grocery shopping receipts for the past 6 weeks and to provide a second 24-hour urine sample at this appointment or within a week of their follow-up visit. The research team will be responsible for arranging follow-up visits. Participants will be contacted by phone in the first instance (unless an alternative contact method is requested by the participant). The research team will use text messages to remind participants of their upcoming appointments.

\section{Accompanied shop}

We will undertake an accompanied grocery shopping trip with a convenience sample of participants (both intervention and control), using the think-aloud method. The aim of this is to understand how the study materials and content influence shopping and wider dietary behaviours, and to assess the barriers to and facilitators of using the Salt Swap app.

Retrospective questioning via a semi-structured interview immediately after the accompanied shop will add depth to the information gathered about the participants' thought processes and understanding of the role of diet in blood pressure control. This qualitative assessment will be undertaken at staggered time points throughout the study.

\section{Randomisation}

Once participants have provided consent and completed the baseline data collection, the research team will randomise them to one of the two trial arms using computergenerated randomisation. The randomisation will be stratified by general practice using block randomisation in blocks of six. A researcher not involved in taking consent or measuring study outcomes will generate the random number sequence using an online random sequence generator (www.sealedenvelope.com) and assign participants to the intervention groups according to the allocation sequence, to ensure allocation is concealed from the rest of the research team until interventions are assigned. Both the research team and participants will be unaware of the treatment allocation prior to consent.

\section{Blinding}

It is not possible to blind the participants, the clinicians delivering the intervention or the research team to treatment group due to the nature of the intervention. The main trial outcomes are progression criteria and process measures, which are unlikely to be influenced by knowledge of treatment allocation. Intervention effectiveness measures comparing trial arms, which would be used as the primary outcome in a future RCT, are objectively measured outcomes and not open to interpretation by researchers.

\section{Primary outcomes}

The primary objective of the trial is to determine whether to progress to a full trial or whether substantial amendments to the trial procedures are required. Based on previous trials of a similar nature we would hope to achieve $80 \%$ for the first two progression criteria. Allowing for the 95\% confidence interval around this point estimate, we have set the minimum criteria for immediate progression at $65 \%$.

Level one: the following criteria must be met to progress to a full trial with minimal amendments to trial procedures:

1. That $65 \%$ of participants attend the follow-up session.

2. Healthcare professionals typically conduct a brief advice session where at least four out of six of the essential intervention elements are present, assessed by comparing the delivered intervention against a checklist of the components required by the protocol, using audio recordings of the consultation.

3. At least $50 \%$ of participants use the app to scan products on at least one occasion by the end of month one. 
Level two: the following criteria must be met to continue to a full trial with substantial amendments to trial procedures:

1. At least $40 \%$ of participants attend the follow-up session.

2. Healthcare professionals typically conduct a brief advice session where at least a mean of three out of six of the essential elements are present.

3. At least $40 \%$ of participants use the app to scan products on at least one occasion by the end of month one.

If level-two criteria are not met, it would not be considered feasible to continue to a full trial with this trial design.

\section{Secondary outcomes and process measures}

The secondary outcomes include both effectiveness and process measures. The trial is not powered to detect significant differences in effectiveness outcomes. A mix of quantitative and qualitative measures will be used to undertake a process evaluation and to inform sample size estimates for a future trial.

\section{Secondary outcomes (effectiveness)}

The secondary outcomes will be:

- Change in salt content of food purchases in the shopping basket, measured as salt in grams per $100 \mathrm{~g}$ (and in grams per megajoule) in the intervention compared to the control group; assessed throughout the study through collection of the nutrient composition of all purchased products.

- Change in salt intake measured through urinary sodium analysis in the intervention compared to the control group; assessed using 24-h urine collection during the baseline period and at the end of the 6-week follow-up appointment. Sodium excretion, reported as millimoles per litre, millimoles per $24 \mathrm{~h}$ and total volume of the urine sample, will be converted to daily salt intake in grams using the equation: $17.1 \mathrm{mmol}$ of sodium $=1 \mathrm{~g}$ salt, and assumes all of the sodium was derived from salt.

- Change in blood pressure (systolic and diastolic) in the intervention compared to the control group; recorded at baseline and at the 6-week follow-up.

\section{Process measures}

The process measures will be:

- Recruitment rates: total recruited and number per practice per month, (including the number invited who were eligible and consented, and the number of recruited participants that completed all baseline assessments and were randomised).

- Acceptability of the intervention delivery. The perspective of the healthcare professionals responsible for delivering the intervention will be captured via interviews, exploring barriers to and facilitators of delivery and their confidence and belief in their capability to deliver the intervention session.

- App use and acceptability; measured quantitatively through within-app automatic recording (e.g. frequency of use, number of products scanned per user, frequency of swaps presented and accepted, use of specific behaviour change functionality such as goal setting), and measured qualitatively through the post-intervention survey and think-aloud accompanied shopping trips.

- Feasibility of data collection: willingness of participants to submit 24-hour urine samples and completeness of shopping data (including completeness of data on the salt content of purchased foods).

- Evidence of contamination; measured as use of the Salt Swap app by the control group.

- Changes in participant knowledge, perceptions and behaviours in relation to salt intake and health, measured qualitatively through participant questionnaires and semi-structured interviews.

\section{Sample size}

This study is a feasibility study and is not powered to detect a significant intervention effect. However, we calculated that a sample size of 40 was sufficient to estimate our progression criteria outcomes within acceptably narrow confidence intervals to enable robust testing of the trial methods. Recruiting 40 patients will enable the anticipated follow-up rate of $80 \%$ to be estimated within $+/-12 \%$ (80\%, 95\% C.I. 68-92).

\section{Planned analysis}

Baseline characteristics will be reported by trial arm using summary descriptive statistics.

\section{Progression criteria}

Descriptive statistics with $95 \%$ confidence intervals will be reported. Progression criteria will use data from all randomised participants.

\section{Effectiveness measures}

The analysis will test the change in urinary sodium and change in blood pressure. These effectiveness outcomes will be analysed on an intention-to-treat basis, using regression with baseline adjustment to calculate difference in means, and we will present these summary statistics with $95 \%$ confidence intervals. We will test for normality 
of the residuals and use the non-parametric MannWhitney $U$ test, if this model is unacceptable. The analysis will test the change in salt content of food purchases from baseline over the 6-week follow-up, using linear mixed-effects regression models to account for repeat observations. Effectiveness analysis will be based on data from all participants for whom both a baseline and a follow-up measure is available. We will examine the sensitivity of the results to confounding due to differences in baseline characteristics. We will also adjust for the number of people in the household.

\section{Process measures}

Descriptive statistics with 95\% confidence intervals will be used to report the process measures. Process measures will be based on all data available, regardless of whether participants completed the trial or withdrew. We propose to use framework analysis, a thematic research method, to analyse the data from the think-aloud shopping sessions and retrospective interviews $[29,30]$.

\section{Adverse events}

We will only provide standard dietary advice consistent with recommendations from scientific advisory committees in the UK and internationally and we do not expect any related adverse events. However, any related, unexpected, serious adverse events (RUSAEs) will be recorded and reported to the Research Ethics Committee in accordance with the Health Research Authority (HRA) process. The duration of the serious adverse event (SAE) recording period lasts from enrolment in the study to the end of the termination of their programme. All RUSAEs will be recorded at the time the research team become aware of the incident.

An SAE is defined as any untoward medical occurrence that:

- Results in death

- Is life-threatening

- Requires inpatient hospitalisation or prolongation of existing hospitalisation

- Results in persistent or significant disability/incapacity

- Consists of a congenital anomaly or birth defect

All study-related SAEs will be included in the final trial report.

\section{Data management}

The trial will be run in accordance with Good Clinical Practice (GCP) and access to data will be restricted to appropriately trained members of the research team and host organisation as necessary for audit and regulatory purposes. Data will be kept in accordance with the Data Protection Act, 1998 (DPA). Participant-identifiable information will be available to the researcher responsible for conducting accompanied grocery shopping visits and follow-up visits, as it is important that these data are known to the researcher. Otherwise, confidentiality will be maintained.

Data will be entered by a trained researcher directly into a secure online database incorporating an electronic case report form (CRF), using a unique participant ID. The database includes data validation checks and, prior to database lock, a dataset review will be undertaken by the chief investigator to ensure all queries have been resolved and the dataset is complete. All participantidentifiable data linked to the participant via their unique ID will be kept separately from anonymised research data in a password-protected file. Audio recordings will only be stored with participant ID in a password-protected file on secure University of Oxford servers. A study-specific data management plan (DMP) will be in place detailing the procedures necessary to ensure that high-quality data are produced for statistical analysis.

\section{Trial management committee}

The Trial Management Committee will comprise the authors of this protocol, along with two members of the public. The chief investigator will be responsible for project coordination, with oversight from the senior coinvestigators. All investigators will be trained in GCP, and will take appropriate actions to safeguard participants and the quality of the trial.

\section{Dissemination}

The results of this research will be published in academic peer-reviewed journals and through presentations at relevant conferences. They will also be communicated to study participants and members of the public involved in the research through email newsletters and press releases targeted to the specific audience. Authors will acknowledge the study funders. Authorship will be determined in accordance with the International Committee of medical Journal Editors (ICMJE) guidelines and other contributors will be acknowledged.

\section{Discussion}

This study will determine the feasibility of a novel, brief intervention to reduce salt intake, and of the trial design and methods to collect outcome measures. The growth of smartphone (and app) use provides an opportunity to develop behaviour change interventions that can reach large numbers of the population. There is an increasing body of evidence that interventions using apps to change dietary behaviour can be effective [31]. Furthermore, interventions encouraging people to swap to healthier products while grocery shopping in-store or online have 
also been shown to have potential for changing purchasing behaviour [32]. This study will add to the evidence base in this area by providing feedback on the acceptability of and engagement with interventions of this nature. It will address an important gap in the literature on how to provide interventions at scale to support people with high blood pressure to reduce their salt intake.

In the field of behaviour change, researchers are increasingly encouraged to report the evaluation of behaviour change interventions using established taxonomies and ontologies [33]. The outcomes included in this trial will show whether participants engage with the intervention as a whole and help us to understand their engagement with the specific behaviour change techniques incorporated within it. The think-aloud grocery shopping sessions and interviews with participants will provide real-world observations on the usability of the intervention. Furthermore, the qualitative and quantitative feedback from healthcare professions will enable us to assess the feasibility of delivering the intervention as part of routine care in primary care. A further strength of the study is the inclusion of clinical outcomes measures, which would be used in a future trial to evaluate clinical effectiveness.

This study utilises a novel method for collection of grocery shopping data, in addition to the more standard method of collecting till receipts. This has the potential for more efficient and robust assessment of nutrient outcomes, and the trial will provide valuable evidence on the feasibility of this method.

The study is limited in its ability to assess the study outcomes among a diverse range of individuals, due to the small sample size and regional recruitment area. Similarly, the small sample size limits the ability to assess the effectiveness of the intervention. The single follow-up assessment at 6 weeks is appropriate for a feasibility-stage trial but a future large-scale trial would include a longer-term follow-up in order to measure sustained effects on behaviour change and the impact on blood pressure. However, if the outcomes indicate the trial is feasible, and there is potential evidence of behavioural change, this study will provide valuable data to inform power calculations for a future trial on the effectiveness in reducing salt intake and lowering blood pressure in a broader study population.

If successful, this intervention approach could be applied not only to people with high blood pressure but also to the wider population with normal blood pressure in whom dietary salt intake exceeds recommendations.

\section{Trial status}

Recruitment of participants began on 25 September 2018 and will be open until May 2019. The current trial protocol is version 2.0 dated 20 July 2018 .

\section{Abbreviations}

app: Smartphone application; BCW: Behaviour Change Wheel; CRF: Case report form; CVD: Cardiovascular disease; DMP: Data management plan; GCP: Good Clinical Practice; GP: General practitioner; HCP: Healthcare professional; HRA: Health Research Authority; ICMJE: International Committee of Medical Journal Editors; ISRCTN: International Standard Randomised Controlled Trials Number; NHS: National Health Service; NICE: National Institute for Health and Care Excellence; RCT: Randomised controlled trial; RUSAE: Related, unexpected, serious adverse event; REC: NHS National Research Ethics Committee; SAE: Serious adverse event; TDF: Theoretical domains framework

\section{Acknowledgements}

We would like to acknowledge the contributions of the Salt Swap focus group, comprising members of the public with high blood pressure, who contributed to the design and development of the study intervention. We also acknowledge and thank the members of the public and patient involvement panel who reviewed and suggested improvements to documents for participants and David Judge of the Nuffield Department of Primary Care Health Sciences at the University of Oxford who helped create and manage the study database. We thank the George Institute for Global Health for allowing us to use the Foodswitch database of grocery products in this reasearch.

\section{Authors' contributions}

All authors contributed to the study design, edited the paper and critically revised and approved the final manuscript.

\section{Funding}

SPR is funded by a British Heart Foundation fellowship and the Medical Research Council.

SJ and PA are NIHR senior investigators and are funded by the National Institute for Health Research (NIHR) Oxford Biomedical Research Centre (BRC) Obesity, Diet and Lifestyle Theme and National Institute for Health Research (NIHR) Collaboration for Leadership in Applied Health Research and Care Oxford at Oxford Health NHS Foundation Trust.

CP's time on this project was funded by the National Institute for Health Research (NIHR) Collaboration for Leadership in Applied Health Research and Care Oxford at Oxford Health NHS Foundation Trust.

MR is funded by the British Heart Foundation (grant number: 006/PSS/CORE/ 2016/OXFORD).

JS is funded by a Wellcome Trust/Royal Society Sir Henry Dale Fellowship (ref: 211182/Z/18/Z) and also receives funding from the NIHR School for Primary Care Research and the NIHR Collaboration for Leadership in Applied Health Research and Care Oxford at Oxford Health NHS Foundation Trust. The project costs are funded by the British Heart Foundation and the National Institute of Health Research Collaboration for Leadership in Applied Health Research and Care Oxford.

\section{Availability of data and materials}

The data are not publicly available as they contain information that could compromise research participant privacy and consent. The data that support the findings of this study are available on reasonable request from the corresponding author (SPR).

\section{Ethics approval and consent to participate}

This study was reviewed and approved on the 17 March 2017 (reference number 17/SC/0098) by the South Central - Hampshire B Research Ethics Committee (REC) and the Health Research Authority (HRA). Appropriate REC and HRA approval will be sought for any protocol amendments; approved amended versions of the protocol and study documents will be communicated to live recruitment sites via the study site file. One amendment has been approved since recruitment began. This amendment, approved on 4 January 2019, included the addition of two participant letter templates not included in the initial application; and minor revision to the participant information sheet and a participant-facing letter template to clarify inclusion criteria and procedures. Informed consent will be obtained from all study participants.

Consent for publication

Not applicable. 


\section{Competing interests}

The authors declare that they have no competing interests.

\section{Author details}

${ }^{1}$ Nuffield Department of Primary Care Health Sciences, University of Oxford Radcliffe Observatory Quarter, Woodstock Road, Oxford OX2 6GG, UK. ${ }^{2}$ Nuffield Department of Population Health, University of Oxford, Richard Doll Building, Old Road Campus, Roosevelt Drive, Headington, Oxford OX3 7LF, UK.

Received: 7 May 2019 Accepted: 30 August 2019

Published online: 11 October 2019

\section{References}

1. Lim SS, Vos T, Flaxman AD, Danaei G, Shibuya K, Adair-Rohani H, et al. A comparative risk assessment of burden of disease and injury attributable to 67 risk factors and risk factor clusters in 21 regions, 1990-2010: a systematic analysis for the Global Burden of Disease Study 2010. Lancet. 2012; 380(9859):2224-60.

2. GBD 2015 Mortality and Causes of Death Collaborators. Global, regional, and national life expectancy, all-cause mortality, and cause-specific mortality for 249 causes of death, 1980-2015: a systematic analysis for the Global Burden of Disease Study 2015. Lancet. 2016:388(10053):1459-544.

3. Kelly CNM, Stanner SA. Diet and cardiovascular disease in the UK: are the messages getting across? Proc Nutr Soc. 2003;62(3):583-9.

4. WHO. WHO The top 10 causes of death. Fact sheet no.310. Geneva: WHO; 2014. http://www.who.int/mediacentre/factsheets/fs310/en/. Accessed 30 May 2018

5. GBD 2015 Risk Factors Collaborators. Global, regional, and national comparative risk assessment of 79 behavioural, environmental and occupational, and metabolic risks or clusters of risks, 1990-2015: a systematic analysis for the Global Burden of Disease Study 2015. Lancet. 2016;388(10053):1659-724.

6. Ezzati M, Lopez AD, Rodgers A, Vander Hoorn S, Murray CJL. Selected major risk factors and global and regional burden of disease. Lancet. 2002; 360(9343):1347-60.

7. NICE. Hypertension - clinical management of primary hypertension in adults. NICE Clin Guidel 127. 2011

8. He FJ, Pombo-Rodrigues S, Macgregor G, He FJ, Pombo-Rodrigues S, Macgregor GA. Salt reduction in England from 2003 to 2011: its relationship to blood pressure, stroke and ischaemic heart disease mortality. BMJ Open. 2014:4(4):e004549.

9. Elliot P, Stamler J, Nichols R, Dyer AR, Stamler R, Kesteloot H, Marmot M. Intersalt revisited: further analyses of 24 hour sodium excretion and blood pressure within and across populations. BMJ. 1996;312(7041):1249-53.

10. Public Health England. National Diet and Nutrition Survey: assessment of dietary sodium adults (19 to 64 years) in England, 2014. London: PHE; 2016.

11. Scientific Advisory Committee Nutrition. Salt and Health. London: PHE; 2003.

12. Stamler R, Stamler J, Gosch FC, Civinelli J, Fishman J, McKeever P, et al. Primary prevention of hypertension by nutritional-hygienic means. Final report of a randomized, controlled trial. JAMA. 1989;262(13):1801-7.

13. The Trials of Hyertension Prevention Group. Effects of weight loss and sodium reduction intervention on blood pressure and hypertension incidence in overweight people with high-normal blood pressure. The Trials of Hypertension Prevention, phase II. The Trials of Hypertension Prevention Collaborative. Arch Intern Med. 1997;157(6):657-67.

14. Sacks FM, Svetkey LP, Vollmer WM, Appel LJ, Bray GA, Harsha D, et al. Effects on blood pressure of reduced dietary sodium and the Dietary Approaches to Stop Hypertension (DASH) diet. DASH-Sodium Collaborative Research Group. N Engl J Med. 2001;344(1):3-10.

15. Hooper L, Bartlett C, Smith GD, Ebrahim S. Systematic review of long term effects of advice to reduce dietary salt in adults. BMJ. 2002;325:1-9.

16. Rees K, Dyakova M, Ward K, Thorogood M, Brunner E. Dietary advice for reducing cardiovascular risk. Cochrane Database Syst Rev. 2013;3:CD002128.

17. Hooper L, Bartlett C, Davey Smith G, Ebrahim S. Advice to reduce dietary salt for prevention of cardiovascular disease (Review). Cochrane Database Syst Rev. 2004;(1). https://doi.org/10.1002/14651858.CD003656.pub2.

18. Stead L, Buitrago D, Preciado N, Sanchez G, Hartmann-boyce J, Lancaster T. Physician advice for smoking cessation (Review). Cochrane Database Syst Rev. 2013;(5). https://doi.org/10.1002/14651858.CD000165.pub4.

19. Kaner EF, Dickinson HO, Beyer FR, Campbell F, Schlesinger C, Heather $\mathrm{N}_{\text {, }}$ et al. Effectivenss of brief alcohol interventions in primary care populations
(Review). Cochrane Database Syst Rev. 2007;(2). https://doi.org/10.1002/ 14651858.CD004148.pub3.

20. Kulick D, Langer RD, Ashley JM, Gans KM, Schlauch K, Feller C. Live well: a practical and effective low-intensity dietary counseling intervention for use in primary care patients with dyslipidemia-a randomized controlled pilot trial. BMC Fam Pract. 2013;14:59.

21. Little P, Kelly J, Barnett J, Dorward M, Margetts B, Warm D. Randomised controlled factorial trial of dietary advice for patients with a single high blood pressure reading in primary care. BMJ. 2004;328(7447):1054.

22. Eyles $H$, McLean R, Neal B, Jiang Y, Doughty RN, McLean $R$, et al. A saltreduction smartphone app supports lower-salt food purchases for people with cardiovascular disease: findings from the SaltSwitch randomised controlled trial. Eur J Prev Cardiol. 2017;24(13):1435-44.

23. Ipjian ML, Johnston CS. Smartphone technology facilitates dietary change in healthy adults. Nutrition. 2017:33:343-7.

24. Michie S, van Stralen MM, West R. The behaviour change wheel: a new method for characterising and designing behaviour change interventions. Implement Sci. 2011;6(1):42.

25. French SD, Green SE, O'Connor DA, McKenzie JE, Francis JJ, Michie S, et al. Developing theory-informed behaviour change interventions to implement evidence into practice: a systematic approach using the Theoretical Domains Framework. Implement Sci. 2012;7(1):38.

26. NHS Choices. Tips for a lower-salt diet. http://www.nhs.uk/livewell/ goodfood/pages/cut-down-salt.aspx. Accessed 14 Aug 2018.

27. BHF. Cut down on salt. 2013. https://www.bhf.org.uk/publications/healthyeating-and-drinking/cut-down-on-salt. Accessed 14 Aug 2018.

28. Department of Health, Food Standards Agency, Llywodraeth Cymru Welsh Government, Food Standards Scotland Inbhe Bidh Alba. Guide to creating a front of pack (FoP) nutrition label for pre-packed products sold through retail outlets. London: Food Standards Agency; 2016.

29. Ritchie J, Spencer L. Qualitative data analysis for applied policy research. In: Bryman A, Burgess RG, editors. Analyzing qualitative data. London: Routledge; 1994. p. 173-94.

30. Gale NK, Heath G, Cameron E, Rashid S, Redwood S. Using the framework method for the analysis of qualitative data in multi-disciplinary health research. BMC Med Res Methodol. 2013;13(1):117.

31. Schoeppe S, Alley S, Van Lippevelde W, Bray NA, Williams SL, Duncan MJ, et al. Efficacy of interventions that use apps to improve diet, physical activity and sedentary behaviour: a systematic review. Int J Behav Nutr Phys Act. 2016;13(1):127.

32. Hartmann-Boyce J, Bianchi F, Piernas C, Riches SP, Frie K, Nourse R, et al. Grocery store interventions to change food purchasing behaviors: a systematic review of randomized controlled trials. Am J Clin Nutr. 2018; 107(6):1004-16.

33. Michie S, West R, Sheals K, Godinho CA. Evaluating the effectiveness of behavior change techniques in health-related behavior: a scoping review of methods used. Transl Behav Med. 2018;8(2):212-24.

\section{Publisher's Note}

Springer Nature remains neutral with regard to jurisdictional claims in published maps and institutional affiliations.

Ready to submit your research? Choose BMC and benefit from:

- fast, convenient online submission

- thorough peer review by experienced researchers in your field

- rapid publication on acceptance

- support for research data, including large and complex data types

- gold Open Access which fosters wider collaboration and increased citations

- maximum visibility for your research: over $100 \mathrm{M}$ website views per year

At $\mathrm{BMC}$, research is always in progress.

Learn more biomedcentral.com/submissions 